\title{
CANADIAN ANAESTHETISTS \$OCIETY
}

MEMBERSHIP LIST, 1963

\section{British Columbia Division}

Dr. J. L. Andrews

1946 Mayfair Dr.

Victoria, B.C.

Dr. M. G. Atnikov

1068 W. 47th Ave.

Vancouver, B.C.

Dr. Robert B. Bambrook

937 W. 19th Ave.

Vancouver 8, B.C.

Dr. G. W. Barnes

8790 Crest Dr.

Burnaby 3, B.C.

Dr. Marion Berry

Ste. 204, 1160 W. 13th Ave.

Vancouver, B.C.

Dr. G. B. Bigelow

1121 Yates St.

Victoria, B.C.

Dr. A. C. Blair

1112 Eyremont Dr., S.S.2

West Vancouver, B.C.

Dr. Norman J. Campbell

5616 Highbury St.

Vancouver 13, B.C.

Dr. J. J. Carroll

1530 W. 37 th Ave.

Vancouver, B.C.

Dr. H. C. Carruthers

3067 Oakdowne

Victoria, B.C.

Dr. Jone Chang

6875 Tisdall St.

Vancouver, B.C.

Dr. F. S. Chu

16 E. Hastings St.

Vancouver 4, B.C.
Dr. Gordon F. Clark

4220 Evergreen

West Vancouver, B.C.

Dr. R. F. Clark

901 Helena St.

Trail, B.C.

Dr. I. M. Clearihue

2445 Landsdowne Rd.

Victoria, B.C.

Dr. Donald R.Collins

Medical Clinic, 5885 Arbutus Ave.

Powell River, B.C.

Dr. B. E. Cragg

2765 Sea View Rd.

Victoria, B.C.

Dr. Lois D. Crawford

620 King George's Way

West Vancouver, B.C.

Dr. Robert E. Creighton

1505 Tolomie St.

Vancouver, B.C.

Dr. LoisE. Davies

829 W. Broadway

Vancouver, B.C.

Dr. Patrick A. Debenham

713 Columbia St.

New Westminster, B.C.

Dr. John W. Dennis

1459 W. 39th Ave.

Vancouver 13, B.C.

Dr. William A. Dodds

4789 Haggart St.

Vancouver, B.C.

Dr. William A. Doll

6750 Tisdall St.

Vancouver 16, B.C. 
Dr. C. W. Duck

2796 Dufferin

Victoria, B.C.

Dr. E. O. DuVernet

8584 Dufferin St.

Vancouver, B.C.

Dr. William L. Esdale

4487 Townley St.

Vancouver 8, B.C.

Dr. Jose Fajnbuch

1145 W. 11th Ave., Ste. 203

Vancouver, B.C.

Dr, Wm. M. Falk

1145 W. 11th Ave.

Vancouver, B.C.

Dr. Evan G. Forseng

6071 Selkirk St.

Vancouver 13, B.C.

Dr. R. A. Fortye

Royal Jubilee Hospital

Victoria, B.C.

Dr. James B. Fulton

1832 Knox Rd.

Vancouver, B.C.

Dr. Gordon J. Fyffe

6480 Cabeldu Cres.

R.R. \#1

North Surrey, B.C.

Dr. Douglas M. Gardiner

4990 Willow St.

Vancouver 13, B.C.

Dr. A. K. Gibbons

1735 W. 38th Ave.

Vancouver 14, B.C.

Dr. James A. Gibson

12559 99th Ave.

North Surrey, B.C.

Dr. H. B. Graves

6016 Alma Rd.

Vancouver, B.C.

Dr. John W. Green 3096 Cadboro Bay Rd.

Victoria, B.C.
Dr. Marion Griffiths

1909 Quilchena Cr.

Vancouver 13, B.C.

Dr. Edwin R. Hall

815 King George's Way

West Vancouver, B.C.

Dr. W. M. Hall

5511 Wallace

Vancouver, B.C.

Dr. John H. Harland

c/o The Underhill Clinic

1635 Abbott St.

Kelowna, B.C.

Dr. Murray C. Hay

3724 Clark Drive

Vancouver 10, B.C.

Dr. Edwin R. Henry 2025 Lansdowne Rd. Victoria, B.C.

Dr. J. M. Hiddleston

Ste. 703, 1025 Gilford

Vancouver 5, B.C.

Dr. Shou-hsin Hsu

319 W. 15th Ave.

Vancouver, B.C.

Dr. W. S. Huckvale

108 Koutenay Ave.

Trail, B.C.

Dr. Peter W. Hudson 4094 W. 33rd Ave.

Vancouver, B.C.

Dr. Edward N. Hughes

2175 Kendal Ave.

Victoria, B.C.

Dr. Jean Hugill

3855 Puget Dr.

Vancouver, B.C.

Dr. John A. Hutchison

Doctors' Residence

Vancouver General Hospital

Vancouver, B.C. 
Dr. Leonard C. Jenkins

Vancouver General Hospital

Vancouver, B.C.

Dr. R. A. Jones

940 King George's Way

West Vancouver, B.C.

Dr. Erna Keddis

P.O. Box 132

Port Alberni, B.C.

Dr. Patrick N. Kenny

1492 W. 45th Ave.

Vancouver 13, B.C.

Dr. Geraldine Kent

475 Gordon Place

West Vancouver, B.C.

Dr. H. A. Kester

6497 Granville St.

Vancouver, B.C.

Dr. Peter W. Klassen

945 Florimond Rd.

Richmond, B.C.

Dr. Barbara P. Kraft

P.O. Box 310

Kitimat, B.C.

Dr. K. W. Langston

3280 Mathers Ave.

West Vancouver, B.C.

Dr. Michael Lattey

3009 31st Ave.

Vernon, B.C.

Dr. R. I. Logan

No. 602, 511 Ash St.

New Westminster, B.C.

Dr. Hugh H. Macartney

100 Bonnymuir Dr.

West Vancouver, B.C.

Dr. M. Watson MacCrostie 3062 S.W. Marine Dr. Vancouver 13, B.C.

Dr. H. B. MacEwen

1215 W. 13th Ave.

Vancouver 9, B.C.
Dr. Doris E. Mackay

7522 Wright St.

Burnaby 3, B.C.

Dr. R. Bruce Maclean

Brickyard Road, R.R. 1

Wellington, B.C.

Dr. William G. Markham

1937 W. 44th Ave.

Vancouver, B.C.

Dr. Maria Mate

4371 Greta St.

S. Burnaby, B.C.

Dr. Donald Matheson

2885 W. 34th Ave.

Vancouver 13, B.C.

Dr. Wm. K. Maxwell

1450 Chestnut St., Apt. 1114

Vancouver 9, B.C.

Dr. F. W. McCaffrey

P.O. Box 2845

Vancouver 3, B.C.

Dr. Alexander D. McDougall

Irving Clinic

P.O. Box 150

Kamloops, B.C.

Dr. John C. McGregor

1366596 th Ave.

North Surrey, B.C.

Dr. Norman McMillen

1961 Allison Rd.

Vancouver, B.C.

Dr. J. A. MeNab

2474 W. 36th Ave.

Vancouver, B.C.

Dr. Lorenzo Meloche 4286 W. 8th Ave.

Vancouver, B.C.

Dr. Irene Edith Millar

Box 4194, Postal Station D

Vancouver 9, B.C.

Dr. R. D. Millar

2175 W. 49th Ave.

Yancouver, B.C. 
Dr. Mary Louise Miller 6200 MacDonald St. Vancouver 13, B.C.

Dr. Robert S. Moir 880 King George's Way West Vancouver, B.C.

Dr. James P. Munroe 306-2910 Alder

Vancouver 9, B.C.

Dr. John E. Nixon 5515 Laburnum St. Vancouver 13, B.C.

Dr. J. P. O'Donnell 4116 W. 8th Ave. Vancouver, B.C.

Dr. Thomas J. O'Neil 1635 Abbott St. Kelowna, B.C.

Dr. John L. Oulton 485 Southborough Dr. West Vancouver, B.C.

Dr. George S. Penrose 1088 W. 14th Ave. Vancouver, B.C.

Dr. Peter B. Percheson 3837 Cypress St.

Vancouver 9, B.C.

Dr. I. C. Poole 1049 Devonshire Cres.

Vancouver, B.C.

Dr. J. Porter * 1645 Commercial Vancouver, B.C.

Dr. Tom E. Powell 1877 Comox St. Vancouver 5, B.C.

Dr. David R. Preston 7992 Yukon St. Vancouver 15, B.E.

Dr. H. S. Randall 4786 W. 2nd Ave. Vancouver, B.C.
Dr. Daniel G. Revell 4441 Shore Way Victoria, B.C.

Dr. Elsie Ritch 3779 Blenheim St. Vancouver, B.C.

Dr. G. L. Robinson 3725 W. 29th Ave. Vancouver, B.C.

Dr. Alice K. Ronan 4509 W. 5th Ave. Vancouver 8, B.C.

Dr. Colin A. Sands 4705 Chancellor Blvd. Vancouver 8, B.C.

Dr. Roy C. Schofer 896 W. 15th Ave. Vancouver 9, B.C.

Dr. Maxwell H. Schultz 563 Glenross

West Vancouver, B.C.

Dr. Robert E. Simpson 1281 W. 32nd Ave. Vancouver, B.C.

Dr. G. E. Sleath 6007 Blenheim St. Vancouver 13, B.C.

Dr. G. Wm. Sleath 2850 Willoughby Ave. Burnaby 3, B.C.

Dr. N. A. Stewart 4149 Cypress Vancouver, B.C.

Dr. John R. Stone 3656 Crestview Rd. Victoria, B.C.

Dr. Peter A. Tannen 3626 W. 22nd Ave. Vancouver 8, B.C. 
Dr. Alun Philip Thomas

Irving Clinic

P.O. Box 150

Kamloops, B.C.

Dr. Norman M. Thornton

Burris Clinic

Kamloops, B.C.

Dr. Robert Hugh Thorp

690 Knockmaroon Rd.

West Vancouver, B.C.

Dr. Thomas N. F. Todd

1027. Government St.

Penticton, B.C.

Dr. Benjamin R. Townsley

4132 Virginia Cres.

North Vancouver, B.C.

Dr. E. James Treloar 4594 W. 5th Ave.

Vancouver 8, B.C.

Dr. Fred Walton

827 Cherry

New Westminster, B.C.

Dr. Eric Webb

1512 Acadia Rd.

Vancouver, B.C.
Dr. Henri Wetselaar

877 No. 1 Rd.

Richmond, B.C.

Dr. R. G. Whitehead

3850 Synod Rd.

Victoria, B.C.

Dr. Floyd G. Williams

1725 Grand Blvd.

North Vancouver, B.C.

Dr. Ivor H. Williams

702 Barnham Rd.

West Vancouver, B.C.

Dr. Ralph S. Woodsworth

4638 Pine Cres.

Vancouver, B.C.

Dr. G. H. Worsley

830 Austin Ave.

Coquitlam,

New Westminster, B.C.

Dr. Maxwell Yates

2351 Landsdowne Rd.

Victoria, B.C.

Dr. Zenon G. Zakvorny

821 W. 10th Ave. Apt. 2

Vancouver, B.C.

\section{Alberta Drviston}

Dr. A. Aeillo

Drumheller Associated Physicians

Drumheller, Alta.

Dr. J. O. Anderson

406 Scarboro Ave.

Calgary, Alta.

Dr. Peter Blake-Knox

The Hospital, R.C.A.F. Station

Cold Lake, Alta.

Dr. Donald F. Cameron

9015 Saskatchewan Dr.

Edmonton, Alta.
Dr. Wm. H. Cochrane

14503-84th Ave.

Edmonton, Alta.

Dr. John B. Collip

11141-91st Ave.

Edmonton, Alta.

Dr. Cauldwell G. Craig 4723-56th St.

Red Deer, Alta.

Dr. Victor E. Crapnell

Haig Clinic

Lethbridge, Alta. 
Dr. H. J. De Jongh

Dept. of Anaesthesia

University Hospital

Edmonton, Alta.

Dr. Edgar G. Diamond

Calgary General Hospital

Calgary, Alta.

Dr. Eric H. Dobbs

724 Madison Ave. S.W.

Calgary, Alta.

Dr. Harry V..Donaldson

64 Rosevale Dr.

Calgary, Alta.

Dr. Richard P. Douglas

3906-4A St. S.W.

Calgary, Alta.

Dr. C. Denzil Elton

11627-78th Ave.

Edmonton, Alta.

Dr. Harold H. England

14616-88th Ave.

Edmonton, Alta.

Dr. John F. Farley

6319-111th Ave.

Edmonton, Alta.

Dr. Frank F. Fish

201-33rd Ave S.W.

Calgary, Alta.

Dr. C. M. Fletcher

8711-120th St.

Edmonton, Alta.

Dr. Norman E. Foster

117 Superior Ave.

Calgary, Alta.

Dr. E. A. Gain

8919-140th St.

Edmonton, Alta.

Dr. Patricia O. Goodhall

85 Cambrian Dr.

Calgary, Alta.

Dr. William Hugh Graham

14420-78th Ave.

Edmonton, Alta.
Dr. J. Martin Hagen

11403-72nd Ave.

Edmonton, Alta.

Di. F. C. Haley

University Hospital

Edmonton, Alta.

Dr. A. S. Hall

Royal Alexandra Hospital

Edmonton, Alta.

Di: John R. Hemstock

234-21st Ave. S.W., Apt. $\$$

Calgary, Alta.

Dr. Carl H. Heuchert

211 Medical Arts Bldg.

Calgary, Alta.

Dr. Z. E. Hoar

8224-98th Ave.

Edmonton, Alta.

Dr. Grant K. Hoe

10327-135th St.

Edmonton, Alta.

Dr. H. M. Hutchinson

10111-138th St.

Edmonton, Alta.

Dr. Masatatsu Ishii 410-4th Ave. S.W.

Calgary, Alta.

Dr. Walter S. Johns

512 Rideau Rd.

Calgary, Alta.

Dr. Frank L. Johnson c/o Campbell Clinic 329-7th St. S.

Lethbridge, Alta.

Dr. Alan M. Keil

2215 Lancing Ave.

Calgary, Alta.

Dr. Ebba Kirkebeck

509-15th Ave. N.E.

Calgary, Alta.

Dr. John H. A. Lawrence 40 Chinook Dr. S.W.

Calgary, Alta. 
Dr. A. M. Leacock

Box 910

Olds, Alta.

Dr. C. M. Learmonth 10322-132nd St.

Edmonton, Alta.

Dr. Leopold Lewis

Medicine Hat Clinic

Medicine Hat, Alta.

Dr. F. E. Lundy 3627-12th St. S.W.

Calgary, Alta.

Dr. J. Alastair MacKay 10344-132nd St.

Edmonton, Alta.

Dr. Hugh R. Macphail 7227-114A St.

Edmonton, Alta.

Dr. John D. Manes

300 Medical Arts Bldg.

Calgary, Alta.

Dr. Robert I. McCalla

9566-83rd St.

Edmonton, Alta.

Dr. John W. R. McIntyre

11110-86th Ave.

Edmonton, Alta.

Dr. A.P. Melgrave

2020 Glasgow Dr.

Calgary, Alta.

Dr. D. J. Moffat

14511 McKenzie Dr.

Edmonton, Alta.

Dr. George T. Moonie 8416-118th St.

Edmonton, Alta.

Dr. G. P. Mores

440 Scarboro Ave.

Calgary, Alta.

Dr. Walter J. Mudry 632-16th Ave. N.W. Calgary, Alta.
Dr. Nestor F. Natalio

Royal Alexandra Hospital

Edmonton, Alta.

Dr. Yuen-Cheong Nip

1723-18th Ave. N.W.

Calgary, Alta.

Dr. N. W. Nix

14016 Valleyview Dr.

Edmonton, Alta.

Dr. A. A. O'Neil

1826-17th St. S.W., Ste. 8

Calgary, Alta.

Dr. Solly G. Paletz

11444-71st Ave.

Edmonton, Alta.

Dr. G. R. C. Palmer

439 Mayor Magrath Dr.

Lethbridge, Alta.

Dr. Frederick L. Pamey

c/o 14450 McQueen Rd., Ste. 12

Edmonton, Alta.

Dr. L. J. Patterson

Parsons Clinic

Red Deer, Alta.

Dr. Richard D. Price

Dept. of Anaesthesia

Calgary General Hospital

Calgary, Alta.

Dr. Rhoda B. Robertson

Box 88

Midnapore, Alta.

Dr. John S. Ruddell

2402-5th Ave. S.

Lethbridge, Alta.

Dr. Joseph J. Scales

General Hospital

Edmonton, Alta.

Dr. R. D. Scragg

10408-116th Ave

Edmonton, Alta. 
Dr. Roy R. Spackman

Cardston

Alta.

Dr. Adraan W. J. Starink

209 Medical Centre

Calgary, Alta.

Dr. John D. St. Clair

14635-93rd Ave.

Edmonton, Alta.

Dr. Orest Stechishin

Dept. of Anaesthesia

General Hospital

Edmonton, Alta.
Dr. Isabel C. M. Stevenson 4524 Grove Hill Rd.

Calgary, Alta.

Dr. R. J. Stewart

15206-76A Ave.

Jasper Place, Alta.

Dr. William Strome

Haig Clinic

Lethbridge, Alta.

Dr. C. Maurice Watson

Royal Alexandra Hospital

Edmonton, Alta.

\section{Saskatchewan Drvision}

Dr. Lauro L. Adevoso

University Hospital

Saskatoon, Sask.

Dr. Pamela J. Alport

P.O. Box 523

Regina, Sask.

Dr. E. Asquith

235 Medical and Dental Bldg.

Regina, Sask.

Dr. Azmy R. Boutros

University Hospital

Saskatoon, Sask.

Dr. M. W. Bowering 1010 McCallum Hill Bldg.

Regina, Sask.

Dr. Chung Ai Chang

579 Vaughan St. W.

Moose Jaw, Sask.

Dr. Thomas A. Christie

1010 McCallum Hill Bldg.

Regina, Sask.

Dr. Edward C. Cockings

Dept. of Anaesthesia

University of Saskatchewan

Saskatoon, Sask.

Dr. Davina M. Cowan

1220 Colony St.

Saskatoon, Sask.
Dr. Jose G. D'Almeida

1010 McCallum Hill Bldg.

Regina, Sask.

Dr. T. V. Darke

1010 McCallum Hill Bldg.

Regina, Sask.

Dr. Lucina R. Dimaculangan

University Hospital

Saskatoon, Sask.

Dr. D. M. Ewart

200 Scott Block

Moose Jaw, Sask.

Dr. B. W. Hargarten

103 Medical Bldg.

217 3rd Ave. N.

Saskatoon, Sask.

Dr. Albert Hudec

606 Medical and Dental BIdg.

Regina, Sask.

Dr. Hugh J. Johnston

202 Scott Block

Moose Jaw, Sask.

Dr. J. D. Keene

378 2nd East

Swift Current, Sask. 
Dr. Christopher J. Kilduff 1518 Preston Ave.

Saskatoon, Sask.

Dr. Glen W. Kinsman

673 University Dr.

Saskatoon, Sask.

Dr. Zbigniew T. Kubinski

1113 Grafton Ave.

Moose Jaw, Sask.

Dr. Violet I. A. Larsen

2845 Athol St.

Regina, Sask.

Dr. George H. Laycraft 1010 McCallum Hill Bldg. Regina, Sask.

Dr. W. B. MacDonald

14 Mitchell St.

Saskatoon, Sask.

Dr. D. F. McAlpine 1010 McCallum Hill Bldg. Regina, Sask.

Dr. Mary A. Nicholson

401 3rd Ave. N.

Saskatoon, Sask.
Dr. Thomas Pimblett

University Hospital

Saskatoon, Sask.

Dr. Rodger F. Pugh

607 Cumberland Ave.

Saskatoon, Sask.

Dr. Emanuele Rapicavoli

University Hospital

Saskatoon, Sask.

Dr. Edith P. Rogoman

1123 Ave. I S.

Saskatoon, Sask.

Dr. Geoffrey C. Ryan

1010 McCallum Hill Bldg.

Regina, Sask.

Dr. Leslie C. Skinner

2701 Regina Ave.

Regina, Sask.

Dr. John B. Trudelle

2936 Regina Ave.

Regina, Sask.

Dr. W. E. Upthegrove

735 University Dr.

Saskatoon, Sask.

Dr. Gordon M. Wyant

522 Copeland Cres.

Saskatoon, Sask.

\section{Manitcba Drviston}

Dr. O. H. Arseven

Interns' Quarter

Winnipeg General Hospital

Winnipeg, Man.

Dr. Dorothy Barnhouse 283 Belvedere St., St. James Winnipeg 12, Man.

Dr. Marjorie Bennett

St. Boniface Hospital

St. Boniface, Man.

Dr. G. V. Bigornia

15 Eastgate

Winnipeg 1, Man.
Dr. Max Cham

148 McAdam Ave.

Winnipeg 4, Man.

Dr. Julio P. Cortes

577 Simcoe St.

Winnipeg 10, Man.

Dr. W. J. Crawford 4611 Roblin Blvd. Charleswood, Man.

Dr. Lionel F. G. Cruickshank

320 Dromore

Winnipeg, Man. 
Dr. John D. Culligan 872 Campbell St. Winnipeg 9, Man.

Dr. James H. Daniels 408 Laidlaw Blvd. Winnipeg 29, Man.

Dr. S. L. Drulak 308 Boreham Blvd. Winnipeg 29, Man.

Dr. Gonopoti Dumo Dept. of Anaesthesia Winnipeg General Hospital Winnipeg, Man.

Dr. Morris Erenberg 784 Ash St.

Winnipeg 9, Man.

Dr. Homer Eshoo

31 Kingston Row

Winnipeg 8, Man.

Dr. Hisashi Furuiye

Dept. of Anaesthesia

Winnipeg General Hospital

Winnipeg 3, Man.

Dr. Myrle A. Gard

812 McDermot Ave.

Winnipeg 3, Man.

Dr. Donald L. Gemmill

Box 580

The Pas, Man.

Dr. K. G. Grant

Ste. 4, Willis Apts.

Norwood, Man.

Dr. Remedios A. Guillermo Winnipeg General Hospital Winnipeg, Man.

Dr. T. Hayakawa 35 Tod Drive Winnipeg 8, Man.

Dr. Arnold W. Holm 1220 Wellington Cres. Winnipeg, Man.

Dr. R. L. Howden 273 Tache Ave. Norwood, Man.
Dr. D. M. H. Huggins

99 Niagara St.

Winnipeg, Man.

Dr. H. C. Hutchison

171 Yale Ave.

Winnipeg, Man.

Dr. Thorberg Johannesson

763 Renfrew St.

Winnipeg, Man.

Dr. Sam Kantor

849 Oak St.

Winnipeg 9, Man.

Dr. Lajos Kovacs

408 Selkirk Ave.

Winnipeg, Man.

Dr. R. Somerville Lambie

727 Somerset Ave., Fort Garry

Winnipeg 19, Man.

Dr. Ma. F. S. Lee

Winnipeg General Hospital

Winnipeg, Man.

Dr. Dixie Lim

St. Boniface Hospital

St. Boniface, Man.

Dr. Robert Lim

75 Frontenac Bay

St. Boniface 6, Man.

Dr. Josephine Lo,

Winnipeg General Hospital

Winnipeg 3, Man.

Dr. Louis. Loban

Ste. 24, Hastings Apts.

2193 Portage Ave.

St. James, Man.

Dr. Margaret Loewen

125 Willowdale Place, N. Kildonan

Winnipeg 16, Man.

Dr. Margaret L. Lu

Winnipeg General Hospital

700 William Ave.

Winnipeg 3, Man.

Dr. Joseph McCammon

176 Old Mill Rd.

Winnipeg 12, Man. 
Dr. Thomas J. McCaughey

Children's Hospital

Winnipeg, Man.

Dr. Patrick M. F. McGarry

4549 Roblin Blvd.

Charleswood, Man.

Dr. Bernadette P. McGivern

Ste. 10, Gloucester Apts.

28 Woodrow Place

Winnipeg, Man.

Dr. Robert W. Milner

169 St. Vital Rd., St. Vital

Winnipeg 8, Man.

Dr. Max Minuck

St. Boniface Hospital

St. Boniface, Man.

Dr. Azarmdokht Movafagh

Ste. 10,805 Sherbrooke St.

Winnipeg, Man.

Dr. A. W. Natsuk

301 Carpathia Rd.

Winnipeg, Man.

Dr. Turgut S. Nese

Winnipeg General Hospital

Winnipeg, Man.

Dr. Seamus O'Brien-Moran 450 3rd St.

Brandon, Man.

Dr. Peter G. Premachuk

Flin Flon, Man.
Dr. Graham M. Ross

1180 Wolseley Ave.

Winnipeg 10, Man.

Dr. Gunther Semelka

412 Kelvin Blyd.

Winnipeg 29, Man.

Dr. Ben Shell

811 Lanark St.

Winnipeg 9, Man.

Dr. Walter B. Syslak

Winnipeg General Hospital

Winnipeg, Man.

Dr. David Tass

395 Elm St.

Winnipeg, Man.

Dr. Helen L. S. Tennent

7B Panama Ct.

785 Dorchester Ave

Winnipeg 9, Man.

Dr. G. N. Willson

Box 160

Flin Flon, Man.

Dr. Christopher Wolkenstein

785 Queenston

Winnipeg 9, Man.

Dr. Chiu Yum-San

Deer Lodge Hospital

Winnipeg 12, Man.

Dr. Wasyl Zaycew

1329 Waller Ave.

Winnipeg 9, Man.

\section{Ontario Division}

Dr. D. C. Aikenhead

5088 Lakeshore Hwy., R.R. 2

Burlington, Ont.

Dr. D. W. Aitken

311 Rubidge St.

Peterborough, Ont.

Dr. Elizabeth Aitken

1083 The Parkway

London, Ont.
Dr. G. W. A. Aitken

Box 49

Grand Bend, Ont.

Dr. Ronald R. Aitken

1083 The Parkway

London, Ont.

Dr. C. L. Anderson

228 King St.

St. Catharines, Ont. 
Dr. Daniel Antonevych 686 Westdale Ct.

Oshawa, Ont.

Dr. H. P. Andry

8 Nancy St.

Hamilton, Ont.

Dr. Willis M. Archibald

24 Farmerbrook Place

Kitchener, Ont.

Dr. George C. Armitage

51 Main St. N.

Brampton, Ont.

Dr. Haine I. Axelrod

31 Hilltop

Toronto, Ont.

Dr. Paul Bailey

98 Bideford Ave.

Downsview, P.O., Ont.

Dr. Zonko Bak

21 Cranleigh Ct.

Islington, Ont.

Dr. Robert S. Baker

33 Baby Point Cres.

Toronto, Ont.

Dr. G. R. Balfour

307 Warren Rd.

Toronto, Ont.

Dr. H. C. Balmer

266 Runnymede Rd.

Toronto, Ont.

Dr. Martin G. Bard 1222 Bonnie Cres.

Ottawa 3, Ont.

Dr. H. A. Barends

303 Fairmont Ave.

Ottawa, Ont.

Dr. O. R. Bartlett

5020 Riverside Dr.

Riverside, Ont.

Dr. Gerald S. Base

298 Woodland Dr.

Oakville, Ont.
Dr. D. C. Bastow

30 Riverview Dr.

Toronto, Ont.

Dr. E. Bateman

18 Brule Gdns.

Toronto, Ont.

Dr. R. G. Bateman

217 Pinnacle Ave.

Belleville, Ont.

Dr. M. V. Bates

1550 Glen Watson Dr.

Orchard Hts.

Port Credit, Ont.

Dr. Kenneth L, Baxter

Box 1240

Bracebridge, Ont.

Dr. Matthew Bazoian

249 Caroline St. S.

Hamilton, Ont.

Dr. Anita C. Beaton

15 Collier St.

Barrie, Ont.

Dr. David N. Bell

2121 Killarney Dr.

Ottawa, Ont.

Dr. Wm. H. Bentham

24 Bendale Blvd.

Scarborough, Ont.

Dr. D. W. S. Best

2066 Blairholm Ave.

Burlington, Ont.

Dr. K. Bhavakula

Toronto General Hospital

Toronto, Ont.

Dr. Mario Bianco

105 Stephen Dr.

Toronto 18 , Ont.

Dr. G. N. Black

550 King St.

Port Colborne, Ont.

Dr. John H. Blair

11 Greenbrook Dr.

Toronto 15, Ont. 
Dr. E. C. Blatchford

99 Hill Cres.

Scarborough, Ont.

Dr. J. A. Blezard

1101 The Parkway

London, Ont.

Dr. G. D. M. Boddington

167 Rosedale Heights Dr.

Toronto, Ont.

Dr. William Bota

2234 King St. E.

Hamilton, Ont.

Dr. Donald E. Boyer

1535 Westbrook Dr.

Peterborough, Ont.

Dr. H. W. Boyes

417 Rosemary Rd.

Toronto, Ont.

Dr. J. H. L. Brennan

68 Fallingbrook Rd.

Toronto, Ont.

Dr. A. T. Brown

4 Wychwood Rd.

St. Catharines, Ont.

Dr. Wm. M. Brummitt

54 Broadpath Rd.

Don Mills, Ont.

Dr. G. C. Bryan

540 Mount Pleasant Rd.

Toronto, Ont.

Dr. W. Butt

7 Conrad Ave.

Toronto, Ont.

Dr. John R. Calder

20 Dundas St.

Brantford, Ont.

Dr. Douglas Y. Caldwell

149 Three Valleys Dr.

Don Mills, Ont.

Dr. W. S. Caldwell

84 Main St. S.

Brampton, Ont.

Dr. Harold Cameron

Dept. of Anaesthesia

Westminster Hospital

London, Ont.
Dr. John D. Cameron

55 Kingsmount Blvd.

Sudbury, Ont.

Dr. A. D. Campbell

165 Queen St. N.

Kitchener, Ont.

Dr. Don L. Campbell

2769 Lundys Lane

Niagara Falls, Ont.

Dr. Michael Campbell

32 Lawrence Cres.

Toronto 12, Ont.

Dr. Stanley MI. Campbell

178 St. George St.

Toronto 5, Ont.

Dr. W. A. Campbell

123 College St.

Kingston, Ont.

Dr. Joseph T. Campolongo

222 Eramosa Rd.

Guelph, Ont.

Dr. Doreen Caplin

32 Forest Wood

Toronto 12, Ont.

Dr. Harriett A. Cawthorpe

Box 1063

Sudbury, Ont.

Dr. Joseph Chaikoff

9 Fairholme Ave.

Toronto 19 , Ont.

Dr. R. A. Chaplin

65 Pepler Ave.

Toronto, Ont.

Dr. Peter A. Charlebois

c/o Scarborough General Hospital

Scarborough, Ont.

Dr. John Chassels

14 Deer Park Cres

Toronto, Ont.

Dr. Kam Chatterjee

309 Crestview Rd.

Faircrest Hts.

Ottawa, Ont. 
Dr. George J. Christ 243 Glasgow St.

Kitchener, Ont.

Dr. P. R. Clancy

52 Green Acres

London, Ont.

Dr. George C. Clark

892 Avenue Rd.

Toronto, Ont.

Dr. Norman S. Clark 110 Milden Hall Rd. Toronto 12, Ont.

Dr. G. F. Clement

2250 Haines Rंd.

Port Credit, Ont.

Dr. Brian H. Coggins

8 Tetbury Cres., Parkway W.

Don Mills, Ont.

Dr. Arthur H. Cole

39 Leland Ave.

Toronto 18, Ont.

Dr. A. E. Conley

75 Pine St. N.

Thorold, Ont.

Dr. A. W. Conn

2615 Lake Shore Blvd. W.

Toronto 14, Ont.

Dr. John Wm. Copeman

126 Colborne St.

Simcoe, Ont.

Dr. Charles P. Crompton

7 I Valecrest Dr.

Islington, Ont. .

Dr. F. A. Cuddy

201 Byran St. S.

Whitby, Ont.

Dr. Francis R. Cullen

18 Wallingford Rd.

Don Mills, Ont.

Dr. Louis V. Cullinas

873 Maitland Ave.

Ottawa 3, Ont.
Dr. John K. H. Culverhouse

1243 North Shore Blvd.

Burlington, Ont.

Dr. R. K. Cumming

22 Lynwood Ave.

Ottawa, Ont.

Dr. E. A. Cummings 990 Woodbine Ave.

Toronto, Ont.

Dr. J. E. Curtis

23 Mapledawn Rd.

Etobicoke, Ont.

Dr. John W. Dales

1580 Camelford Rd.

Clarkson, Ont.

Dr. M. Susan Daniel

28 St. Andrews Gdns.

Toronto, Ont.

Dr. J. W. Davidson

15 Limerick Ave.

Toronto 15, Ont.

Dr. Frederick T. Dennis

187 Arthur St.

Port Arthur, Ont.

Dr. W. Derbyshire

70 Princess St.

Fort Erie, Ont.

Dr. H. E. Devlin

15 Liscombe Rd.

Toronto 15, Ont.

Dr. Michael J. Diamond 1061 Don Mills Rd. Don Mills, Ont.

Dr. Jeremiah Dillon

117 King St. E.

Oshawe, Ont.

Dr. G. Wesley Dix

117 Laurentide Dr.

Don Mills, Ont.

Dr. G. D. Dixon -

92 Inglewood Dr.

Toronto, Ont. 
Dr. John J. F. Donworth

28 Montebello Place

St. Catharines, Ont.

Dr. J. Armand Dufresne 785 Montreal Rd.

Ottawa, Ont.

Dr. Francis A. Duggan

2056 Chalmers Rd.

Ottawa 1, Ont.

Dr. Arthur J. Dunn

20 Rumsey Rd.

Toronto, Ont.

Dr. Donald H. Dunn

1189 Queen Victoria Ave.

Lorne Park, Port Credit, Ont.

Dr. Jean E. Dupuy

9 Alexander, Rothwell Hts.

Box 170, R.R. 1

Ottawa, Ont.

Dr. Juliette Durocher

Box 276, R.R. 1

Ottawa, Ont.

Dr. W. O. Moore Ede

102 Kilbarry Rd.

Toronto 7, Ont.

Col. L. H. Edwards

National Defense Medical Centre

Ottawa, Ont.

Dr. Saul M. Eisen

105 Dewboume Ave.

Toronto 10, Ont.

Dr. Clarence C. Evans

$147 \mathrm{McNaughton}$ St.

Sudbury, Ont.

Dr. S. J. Evelyn

377 Vaughan Rd.

Toronto, Ont.

Dr. H. Barrie Fairley

17 Strathallen Blvd.

Toronto 12, Ont.

Dr. Nicholas Farkas

3945 Tecumseh Rd. E.

Windsor, Ont.
Dr. William Farrell

109 West 5 th

Hamilton, Ont.

Dr. Anne Marie Fehrmann

Apt. 1417, City Park Apts.

484 Church St.

Toronto 5, Ont.

Dr. F. C. Ferguson

776 Queens Ave.

London, Ont.

Dr. R. H. Ferguson

5 Bayswater Place

Ottawa, Ont.

Dr. P. W. Ferris

60 De Vere Gdns.

Toronto 12, Ont.

Dr. Donald C. Finlayson

St. Michael's Hospital

Toronto, Ont.

Dr. John E. Fox

3000 Lawrence Ave. E.

Scarborough, Ont.

Dr. R. J. Fraser

125 South Oval St

Hamilton, Ont.

Dr. Gertrude Freund

31 Alexander St., Apt. 1214

Toronto, Ont.

Dr. I. W. Fyfe

30 Lambton Ct.

Sudbury, Ont.

Dr. Anthony Gagliano

1597 Mississauga Rd. W.

R.R. 2, Port Credit, Ont.

Dr. J. M. Boyd Garland

P.O. Box 631

Brantford, Ont.

Dr. Gordon D. Glennie

405 Victoria St.

London, Ont.

Dr. Shashi B. Goel

20 Nuffield Dr.

Scarborough, Ont. 
Dr. R. A. Gordon 178 St. George St. Toronto 5, Ont.

Dr. J. E. Gorman 342 Rosedale Blvd. Windsor, Ont.

Dr. John E. Green 475 Piccadilly Ave. Ottawa 3, Ont.

Dr. H. J. Grennell 50 Gore St.

Kingston, Ont.

Dr. I. C. M. Grimshaw 70 Vaughan Rd.

Toronto, Ont.

Dr. J. M. Growse 219 Wortley Rd. London, Ont.

Dr. Ona-Danute Gustainis 2973 Lawrence Ave. E. Scarborough, Ont.

Dr. T. J. Halkiewicz 37 Duncombe Blvd. Scarborough, Ont.

Dr. Bruce Halliday Box 249

Tavistock, Ont.

Dr. L. M. Hampson 42 Hereford Place Ottawa, Ont.

Dr. E. G. S. Hanley 170 St. George St.

Toronto, Ont.

Dr. T. R. Hanley

70 Heath St. W., Apt. 102

Toronto, Ont.

Dr. Ralph Hargrave 693 McCowan's Rd. Scarborough, Ont.

Dr. Ian Harper 6 Glenelia Ave. Willowdale, Ont.
Dr. Melbourne Harvey

14 Dundana Ave.

Dundas, Ont.

Dr. William Harvey 463-11th St. "B" W

Owen Sound, Ont.

Dr. Kurt Hellman 25 Forest Ridge Dr. Toronto 19, Ont.

Dr. J. S. Heron 4 Spinney Ct. Don Mills, Ont.

Dr. Lewis Hersey 1563 McClure Dr. R.R. 5

London, Ont.

Dr. A. F. Hildebrand 4287 Dufferin St. Downsview, Ont.

Dr. N.P. Hill

73 Welland Ave.

St. Catharines, Ont.

Dr. R. H. Holbrook

24 Undermount Ave. Hamilton, Ont.

Dr. R. L. Hooper 1354 Glenwood Dr. Port Credit, Ont.

Dr. Gordon M. Horne Apt. 701

1300 York Mills Rd.

Don Mills, Ont.

Dr. G. Robert Horne

50 Gore St.

Kingston, Ont.

Dr. J. H. Howell

140 Blair Rd.

Galt, Ont.

Dr. W. Howitt 115 Woolwich St. Guelph, Ont.

Dr. Martin H. Humphrys

Church Street

St. Mary's, Ont. 
Dr. Douglas D. Hunter

3 Swindon Rd.

Islington, Ont.

Dr. Mary E. Hunter

51 Alexander St., Apt. 704

Toronto, Ont.

Dr. Nancy K. Ironside

7 Shorncliffe

Toronto, Ont.

Dr. E. J. Jackson

77 Rochester

Toronto, Ont.

Dr. J. R. Jacobsen

144 Hilton Ave.

Toronto, Ont.

Dr. Wm. A. Jamës

4000 Yonge St.

Apt. 121

Toronto, Ont.

Dr. Gesina E. H. Janssen

50 Selkirk St., Apt. 403

Ottawa, Ont.

Dr. William R. Johns

Glasgow Rd.

R.R. 4

Kitchener, Ont.

Dr. W. S. Johnston

361 Dufferin Ave.

London, Ont.

Dr. Wm. M. Jones

90 Heath St. W., Apt. 104

Toronto 7, Ont.

Dr. Anna Josipowicz

17 Markdale Ave.

Toronto 10, Ont.

Dr. H. T. Jost

267 O'Connor St.

Ottawa, Ont.

Dr. F. L. Junkin

311 Walmer Rd.

Toronto, Ont.

Dr. Brigitte D. Kalow

53 Douglas Cres.

Toronto 5, Ont.
Dr. Harold T. Kay

22 Sunset Blvd.

Ottawa, Ont.

Dr. Marvin Kay

15 Warwick Ave.

Toronto 10, Ont.

Dr. Hubert B. Keenleyside

104 Elworthy Ave.

London, Ont.

Dr. W. Elliott Keith

2450 Lincoln Rd.

Windsor, Ont.

Dr. M. J. Kelly

360 Sixth Ave.

Timmins, Ont.

Dr. James H. Kerr

166 Three Valleys Dr., Parkway W.

Don Mills, Ont.

Dr. Robert M. Kilborn

174 Lydia St.

Kitchener, Ont.

Dr. Ralph John Kimmerly

152 Simcoe St. N.

Oshawa, Ont.

Dr. C. M. Kincaide

57 Glenrose Ave.

Toronto 7, Ont.

Dr. F. A. Kinnaird

Russell, Ont.

Dr. R. S. Kneeshaw

5 Hilldown Tree Rd.

Islington, ${ }^{2}$ Ont.

Dr. V. H. Korth

7 Southlawn Dr.

Agincourt, Ont.

Dr. K. A. Kraft

180 Pineland Ave.

Burlington, Ont.

Dr. Maria Kryt

355 Castlefield Ave.

Toronto 12, Ont.

Dr. J. N. Kyles

90 Melrose Ave. S.

Hamilton, Ont. 
Dr. Douglas T. Law

Box 567, R.R. 1

Ottawa, Ont.

Dr. H. H. Lees

1493 Wyandotte St. E.

Windsor, Ont.

Dr. Blois C. LePage

22 Jason $\mathrm{Rd}$.

Rexdale, Ont.

Dr, Marcus H. Little

53 Silver St.

Kingston, Ont.

Dr. Hubert H. Lloyd

34 Beckwith S.

Smiths Falls, Ont.

Dr. J. R. Loudoun

85 Woolwich St.

Guelph, Ont.

Dr. Jeva Rogers Loughead

561 Broadview Ave.

Toronto, Ont.

Dr. G. H. W. Lucas

Dept. of Pharmacology

University of Toronto

Toronto, Ont.

Dr. Maria E. Lukowski

90 Castleknock Rd.

Toronto 12, Ont.

Dr. C. W. P. Lunderville

48 Broadpath

Don Mills, Ont.

Dr. Ahti S. M. Lundquist

878 Kawartha Dr.

Peterborough; Ont.

Dr. J. D. C. MacDonald

906 Lake Shore Rd.

Sarnia, Ont.

Dr. James F. MacIntosh

911 Wellington St. E.

Sault Ste. Marie, Ont.

Dr. Iain M. MacKay

343 Belsize Dr.

Toronto 12, Ont.
Dr. R. G. Mackenzie 193 Island Park Dr.

Ottawa, Ont.

Dr. A. M. Mackinnon

153A Norfolk St.

Guelph, Ont.

Dr. Edward L. Magee

311 Rubidge St.

Peterborough, Ont.

Dr. James H. S. Mahood

29 Hillcroft Dr.

Kingston, Ont.

Dr. Maurice Mainville

Box 407, R.R. 1

Ottawa, Ont.

Dr. I. W. Mann

442 N. Christina St.

Sarnia, Ont.

Dr. Peter Marchant

56 Pine Cres.

Toronto, Ont.

Dr. L. Paul Marier

723 Trojan

Ottawa, Ont.

Dr. Brian M. Marshall

17 Cornish Rd.

Toronto 7, Ont.

Dr. W. E. Martin

Torrance, Ont.

Dr. Donald I. Matheson

12 Glenwood Cres.

Toronto'16, Ont.

Dr. R. L. Matthews

Croydon Towers, Apt. 1006

19 Lascelles Blvd.

Toronto 7, Ont.

Dr. Philip C. McCabe

233 Coronado Blvd.

Tecumseh, Ont.

Dr. A. B. McCarter

126 Norfolk St.

Guelph, Ont. 
Dr. Thomas McConnachie

38 Highcliffe Ave.

Hamilton, Ont.

Dr. J. E. McCutcheon

25 Summit Ave.

Port Arthur, Ont.

Dr. Richard M. McCutcheon

20 Barrie St.

Kingston, Ont.

Dr. John W. McDougall

35 Garden St.

Brockville, Ont.

Dr. Wilfred B. McGuire

132 Norfolk St. S.

Simcoe, Ont.

Dr. Ronald W. McIntosh 470 Mortimer Ave., Apt. 304

Toronto, Ont.

Dr. Donald H. McIntyre

123 Welland Avè.

Toronto 7, Ont.

Dr. Sheilah McLachlin

437 Highland Rd.

London, Ont.

Dr. Kenneth William McLandress

P.O. Box 639

Mitchell, Ont.

Dr. Donald J. McLean

Ste. 201, 300 King St. W.

Oshawa, Ont.

Colonel N. H. McNally

Headquarters Central Command

Ortona Barracks

Oakville, Ont.

Dr. Murray Mendelson

c/o 602 Castlefield Ave.

Toronto, Ont.

Dr. R. H. Meredith

82 Old Mill Rd.

Toronto 18, Ont.

Dr. Michael W. Michalkoff

621 Cummings Ave., Apt. 211

Ottawa, Ont.
Dr. A. S. Middlebro

138 Tenth St. W.

Owen Sound, Ont.

Dr. Agnes Moffat

327 Charlotte St.

Peterborough, Ont.

Dr. Mayhew G. Moore

118 John Blvd.

Waterloo, Ont.

Dr. John H. Moran

312 Sunnyside Ave.

Toronto, Ont.

Dr. Deane K. Morgan 183 James St. S.

Hamilton, Ont.

Dr. A. G. Morris

536 Wellington St.

London, Ont.

Dr. M. L. Morris

118 Simcoe St. N.

Oshawa, Ont.

Dr. M. Vivyan Morton

180 Manor Rd.

Rockcliffe

Ottawa, Ont.

Dr. H. E. Mountain

Chatsworth, Ont.

Dr. Gladys Munroe

391 Galt Ave.

Oakville, Ont.

Dr. James G. Murray

21 Alpaca Dr.

Scarborough, Ont.

Dr. Saeko S. Nabeta

Toronto General Hospital

Toronto, Ont.

Dr. Raymond Neill

The Spence Clinic

910 Ridgeway St.

Fort William, Ont.

Dr. H. H. Neily

Box 236

Collins Bay, Ont.

Dr. Valentinas A. Nekus

34 Hillcroft Dr.

Kingston, Ont. 
Dr. O. R. Newton

P.O. Box 446

Strathroy, Ont.

Dr. H. R. Nicklin

73 Erie St. S.

Leamington, Ont.

Dr. John S. Nielsen

Ste. 211, Medical-Dental Bldg.

450 Central Ave.

London, Ont.

Dr. Ayao Noguchi

2 Redbank Rd.

Toronto 15, Ont.

Dr. Harry T. Norry

3 Mountfield Dr.

London, Ont.

Dr. Rudolph F. Ohlke

2076 Harvest Dr.

Cooksville, Ont.

Dr. Elizabeth A. Oliver

51 Alexander St., Apt. 1001

Toronto 5, Ont.

Dr. Robert Orange

205 Wembley Dr.

Sudbury, Ont.

Dr. Kathleen S. O'Reilly

63 Sir William's Lane

Islington, Ont.

Dr. Stephen J. O'Rourke

27 Denewood Cres.

Don Mills, Ont.

Dr. T. H. Orton

153 Simcoe St. N.

Oshawd, Ont.

Dr. John S. Packham

325 Victoria Ave.

Chatham, Ont.

Dr. Norman S. Park

282 Bessborough Dr.

Toronto 17, Ont.

Dr. Ellice A. Parmenter 108 Glenvale Blvd.

Toronto 17, Ont.
Dr. John C. Parry

574 King St. W.

Chatham, Ont.

Dr. J. A. Peart

33 White St.

St. Thomas, Ont.

Dr. David A. Pelton

575 Glengrove Ave. W.

Toronto 19, Ont.

Dr. P. Arcade Perrier

511 Main E.

Hawkesbury, Ont.

Dr. V. L. Politi

976 Brucedale Ave. E.

Hamilton, Ont.

Dr. J. B. Poole

417 Dundas St.

Woodstock, Ont.

Dr. Allan S. Porter

187 Arthur St.

Port Arthur, Ont.

Dr. Joan B. Prelipp

658 Sequin Cres.

Port Credit, Ont.

Dr. Ralph I. Probert

17 Mount Royal Ave.

Hamilton, Ont.

Dr. A. L. Purdon

165 Plymouth Rd.

Welland, Ont.

Dr. Ivan Rado

325A James St. S.

Hamilton, Ont.

Dr. Edgar Rae

5 Mallory Gdns.

Toronto, Ont.

Dr. Eric D. Rathbone

100 Medical Arts Bldg.

Fort William, Ont.

Dr. Benjamin Raxlen

c/o The Doctors' Hospital

45 Brunswick Ave.

Toronto, Ont. 
Dr. Norris H. Ready 809 Donlevie Ave.

Ottawa 3, Ont.

Dr. W. J. Reid 274 King St, W. Chatham, Ont.

Dr. J. W. Reingwertz 73 Brookview Dr. Toronto 19, Ont.

Dr. E. E. Reinke Pembroke, Ont.

Dr. W. G. Reive 178 Claremont Ave. Kitchener, Ont.

Dr. Alexander Relle 30 Knightwood Rd. Toronto, Ont.

Dr. W. A. Renwick 114 Elvaston Dr. Toronto 16, Ont.

Dr. K. R. Richardson 239 Ridout St. S. London, Ont.

Dr. A. R. Riddell 84 Homewood Ave. Kitchener, Ont.

Dr. C. H. Robson 96 Chatsworth Dr. Toronto, Ont.

Dr. J. G. Robinson 43 Greenbrook Dr. Toronto 9, Ont.

Dr. L. E. Roche 3 Clemow Ave., Apt. 3 Ottawa, Ont.

Dr. N. Rosen 327 Palmerston Blvd. Toronto, Ont.

Dr. Jean-Paul Rossignol 663 Portage

Ottawa, Ont.
Dr. Helen M. Rossiter 504 Colborne St.

London, Ont.

Dr. F. Rossitter

186 Wright Ave.

Toronto, Ont.

Dr. Manie Rotenberg

26 Farrington Dr.

Willowdale, Ont.

Dr. C. R. Rutdolph

118 West Colborne St.

Brantford, Ont.

Dr. F. G. Ruston

44 Beulah Ave.

Hamilton, Ont.

Dr. P. A. Sarjeant

674 Broadway Ave.

Toronto, Ont.

Dr. Otto W. Schuh

252 Delamere Ave.

Stratford, Ont.

Dr. Normał A. Scott

311 Rubidge St.

Peterborough, Ont.

Dr. Frank L. Seleny

71 Grand Ave., Apt. 306

London, Ont.

Dr. B. A. Seymour

9 Lytton-Blvd.

Toronto, Ont.

Dr. J. M. Shapley

189 Dawlish Ave.

Toronto, Ont.

Dr. Margaret Sheehan

11 Rainham Place

Don Mills, Ont.

Dr. Edward I. Sheffman

79 Chelford Rd.

Don Mills, Ont.

Dr. H. J. Shields

744 Duplex Ave.

Toronto, Ont. 
Dr. Henry J. Shykoff 1097 Victoria Park Ave.

Toronto, Ont.

Dr. Leif E. Simonsen

79 Winnipeg Ave.

Port Arthur, Ont.

Dr. Alan W. Simpson

45 Lilac St.

Kitchener, Ont.

Dr. Sandra J. Sims

51 Alexander St., Apt. 115

Toronto 5, Ont.

Dr. Paul Ellis Slater

197 Duchess Ave.

London, Ont.

Dr. I. A. Sloan

10 Campbell Cres.

Willowdale, Ont.

Dr. Roman L. Sluzar

2070 Annapolis Ave., Applewood Acres

Cooksville, Ont.

Dr. James Small

306 King St.

Midland, Ont.

Dr. Code Smith

20 Heathcote Ave.

Willowdale, Ont.

Dr. G. Foster Smith

48 Bennington Heights Dr.

Toronto, Ont.

Dr. F. Van V. Snell

66 Binscarth Rd.

Toronto, Ont.

Dr. Alvin R. Spector

2700 Bathurst St., Apt. 211

Toronto 19, Ont.

Dr. Wolfgang E. Spoerel

459 Coombs Ave.

London, Ont.

Dr. J. R. Sproule

Rideway Clinic

Fort William, Ont.
Dr. Natalie M. Staron

58 Greenbrook Dr.

Toronto, Ont.

Dr. C. A. Stewart

1070 Lombardo Ave.|

London, Ont.

Dr. R. M. Stringer

124 Chedoke Ave.

Hamilton, Ont.

Dr. Alicja J. Strzalkowska

31 Alexander St., Apt. 516

Toronto 5, Ont.

Dr. C. E. Sylvester

18 Brunswick St.

Stratford, Ont.

Dr. J. F. Tench

2035 Malbrook Rd.

Oakville, Ont.

Dr. Tuevo Tenhumen

1865 Paris St.

Sudbury, Ont.

Dr. C. J. Thiel

National Defence Medical [Centre

Ottawa, Ont.

Dr. Murray C. Thompson

7 Windy Ridge Dr.

Scarborough, Ont.

Dr. John H. Thorn

22 Thorncliffe Pk. Dr., Apt. 203

Toronto 17, Ont.

Dr. C. E. Tipping

1 Brentwood Rd. N.

Toronto 18, Ont.

Dr. Albert A. Toms

3 Bilston Ct.

Toronto 18, Ont.

Dr. J. K. W. Tyl

197 Newton Dr.

Willowdale, Ont.

Dr. N. B. Urie

2400 Rosewood Ave.

Ottawa 13, Ont. 
Dr. Isaac N. Uy, Jr.

Sunnybrook Hospital

Toronto, Ont.

Dr. Jack R. Vanderburgh

200 Cosburn Ave., Apt. 601

Toronto 6, Ont.

Dr. Stuart L. Vandewater

Lake Land Point

Kingston, Ont.

Dr. J. A. Vining

129 Burbank Dr.

Willowdale, Ont.

Dr. F. W. Walker

75 King Edward Ave., Apt. 1

London, Ont.

Dr. Sallie J. Wallace

Department of Anaesthesia

Toronto General Hospital

Toronto, Ont.

Dr. Herbert B. Wallis

290 Wellington

Sarnia, Ont.

Dr. G. Owen Warr

138 Kerr St. N.

Oakville, Ont.

Dr. D. A. Warren

221 Jackson St. W.

Hamilton, Ont.

Dr. W. G. Webster

31 Southwick St.

St. Thomas, Ont.

Dr. R. Leonard Weston

105 Anndale Dr.

Willowdale, Ont.

Dr. J. S. Whalen

94 Princess Anne Cres.

Islington, Ont.

Dr. D. W. Whyte

495 Piccadilly Ave.

Ottawa, Ont.

Dr. A. R. Wilkins

170 St. George St.

Toronto, Ont.
Dr. Ralph D. Williamson

7A Ardaven Place

London, Ont.

Dr. Marta-Maret Wilo

357 Ellis Park Rd.

Toronto 3, Ont.

Dr. Kenneth B. Wilson 25 Westmoreland Rd. Kingston, Ont.

Dr. Willard W. Wilson 16 Cavalier Cres.

Thornhill, Ont.

Dr. V. L. Wincott

305 Sarah St., Box 400

Chippawa, Ont.

Dr. Jules Winemaker 3590 Church St.

Windsor, Ont.

Dr. H. Winesanker 459 Bloor St. W.

Toronto 4, Ont.

Dr. James M. Wishart 630 Bolivar St.

Peterborough, Ont.

Dr. Thomas H. Witton

3014 Eva Dr.

Burlington, Ont.

Dr. G. C. Wray

15 London St. S.

Hamilton, Ont.

Dr. Frederick J. Wright

Kingston General Hospital

Kingston, Ont.

Dr. H. A. Wrong

2180 Main St.

Niagara Falls, Ont.

Dr. Felix Wuerth

6 Forest Hill Dr.

Guelph, Ont. 
Dr. Ming Yao

35 Fern Ave.

Ottawa, Ont.

Dr. Yin-ching Yeung

253 Simcoe St.

Oshawa, Ont.

Dr. J. E. York

36 Astley Ave.

Toronto 5, Ont.
Dr. John M. Yoshioka

369 Morrison Rd.

Oakville, Ont.

Dr. Stanley T. Zeglen

16 Stormont Ave.

Toronto, Ont.

Dr. B. G. Zielonka

7 Woodland Hts.

Toronto 3, Ont.

\section{Quebec Division}

Dr. Jean Albert

2250 Blvd. Laviolette

Trois Rivières, P.Q.

Dr. Eugene Allard

1583 St. Cyrille W.

Quebec, P.Q.

Dr. Raymond Allard

10137 Garnier

Montreal, P.Q.

Dr. Marc Allen

1005 St. Prospere

Trois Rivières, P.Q.

Dr. Jean Archambault

37 Thornton

Montreal, P.Q.

Dr. John Atkinson

349 Graham Blvd.

Town of Mount Royal

Montreal, P.Q.

Dr. Ubald Aubry

320 Marie-Chretienne

Boucherville, P.Q.

Dr. Joseph Aubut

Lac Megantic

Frontenac, P.Q.

Dr. Jacques Audet

5130 Boul. Pie IX, no. 4

Montreal, P.Q.

Dr. Rene Auger,

Jeffery Hale's Hospital

Quebec, P.Q.

Dr. J. E. Barbeau

700 Crémazie E.

Montreal, P.Q.
Dr. Pierre-Paul Barry

5691 Place Donat

Ville D'Anjou

Montreal, P.Q.

Dr. Denis Bazinet

8528 Centrale

Ville Lasalle, P.Q.

Dr. A. Beaudet

1816 Blvd. Laird

Mount Royal, P.Q.

Dr. Pierre P. Bedard

2075-25ième rue

Quebec 3, P.Q.

Dr. Jacques Belanger

2498 Chemin Ste. Foy

Quebec, P.Q.

Dr. Jazeps T. Beldavs

6735 Lemay St., Rosemont

Montreal, P.Q.

Dr. Léo Benoit

585 Ave. Mondor

Ste. Hyacinthe, P.Q.

Dr. Jean-Maurice Bergeron

1262-5th Ave., Shawinigan S.

Montreal, P.Q.

Dr. Bedi S. Berker

$706 \mathrm{Ch}$. Sydenham

Chicoutimi, P.Q.

Dr. Jogues Berthiaume

3220 Louis-Dantin

Montreal 9, P.Q.

Dr. Pauline Biron-Beaudry

P.O. Box 97, Delorimier Station

Montreal 34, P.Q. 
Dr. Lise Bisson

389 Boul. de Normandie

Longueuil, P.Q.

Dr. Yvon Boissonneault

97 St. Theophile

Courville

Quebec 5, P.Q.

Dr. Michel Boisvert

a/s Hôpital St. Ambroise

Loretteville, P.Q.

Dr. Jean-Louis Boivin

2967 de Francheville

Trois Rivières, P.Q.

Dr. A. Bonin

4074 Blvd. Lasalle

Verdun, P.Q.

Dr. Robert R. Boright

4068 Grey Ave.

Montreal 28, P.Q.

Dr. Toussaint Bouchard

195 Bryant N.

Sherbrooke, P.Q.

Dr. Marcel H. Boucher

64 des Chenes-ouest

Quebec, P.Q.

Dr. Marcel A. Boudreau

203 St. Jean Baptiste

Rimouski, P.Q.

Dr. Marcel Boulanger

752 Bloomfield

Montreal 8, P.Q.

Dr. W. Bourne

2165 Lincoln Ave., Apt. 18

Montreal, P.Q.

Dr. Douglas L. Boyd

5550 Cote St. Luc, Apt. 5

Montreal, P.Q.

Dr. J. Maurice Boyer

50 Jacques Cartier

Valleyfield, P.Q.
Dr. Victor Brassard

5018 ième Ave.

Grand'Mere, P.Q.

Dr. G. F. Brindle

454 Stannock Ave.

Town of Mount Royal

Montreal 16, P.Q.

Dr. Philip R. Bromage

24 Fieldfair Ave.

Beaurepaire, P.Q.

Dr. Jean-Louis Brunet

405 Woodlea

Montreal 16, P.Q.

Dr. Adolphe Byk

3400 Bohle

Montreal $\mid 9$, P.Q.

Dr. David Cape

661 Gables Ct.

Beaconsfield, P Q.

Dr. Gaston Carignan

10595 Hamel

Montreal 12, P.Q.

Dr. Jean Caron

2944 Grondines

Ste-Foy, Quebec 10, P.Q.

Dr. Paul Caron

195 Sociale

Quebec 8, P.Q.

Dr. Therese D. Cartier

769 Dunlop

Outremont, P.Q.

Dr. Marcelo Y. Cayetano

2765 DarTing St.

Montreal 4, P.Q.

Dr. Avedis Cetin

2635 Boulvard Pie IX, Apt. 23

Montreal 4, P.Q.

Dr. Roger Chapados

5275 rue Bellechasse, app. 3

Montreal, P.Q.

Dr. M. C. Cheng

132 Sheraton Dr.

Montreal West, P.Q. 
Dr. Georges A. Chevrette 554 Blvd. Manseau

Joliette, P.Q.

Dr. Jean Chouinard

8755 lère Ave.

Charlesbourg 7, P.Q.

Dr. W. J. Ciprick

575-37th Ave.

Lachine West, P.Q.

Dr. Miodrag Cirkovic

9233 Blvd. LaSalle, Apt. 5

Ville LaSalle, P.Q.

Dr. Marcel Clavet

419 Ave. Chantal

Beauport, Quebec 5, P.Q.

Dr. Moise Clermont

3511 Ave. Girouard

Montreal, P.Q.

Dr. Francois Cliche

St-Georges-Ouest, C.P. 279

Cté Beauce, P.Q.

Dr. Max Cohen

4975 Lacombe Ave.

Montreal 26, P.Q.

Dr. Gaston Comtois

190 Blvd. St. Sacrement

Chicoutimi, P.Q.

Dr. Branislava M. Cop-Hodjera

1275 Cote Vertue

Ville St. Laurent, P.Q.

Dr. Gilles Cossette

Hôpital Notre-Dame

Service d'Anesthesie

Montreal, P.Q.

Dr. George Courchesne

1280 rue de Callieres

Quebec, P.Q.

Dr. George Cousineau

5700 Gouin Blyd. W.

Montreal, P.Q.

Dr. Tean-Paul Couture

.17 Charles Edouar

Levis, P.Q.
Dr. Andre Crepeau

10180 rue Meilleur, Apt. 4

Montreal, P.Q.

Dr. John C. Cronhelm

407 Metcalfe Ave.

Westmount, P.Q.

Dr. D. E. Crowell

5768 Glenarden

Montreal 29, P.Q.

Dr. W. G. Cullen

226 Ballantyne Ave. N.

Montreal, P.Q.

Dr. H. T. Davenport

Montreal Children's Hospital

Montreal, P.Q.

Dr. P. Decary

2852 St. Joseph Blvd. E.

Montreal, P.Q.

Dr. Jean-Paul Dechêne

28 ave. de la Colline

Ste Foy, P.Q.

Dr. Andree Delisle

80 Pine ouest, App. 304

Montreal, P.Q.

Dr. Rosario Denis

5625 des Chenes Ave.

Montreal 36, P.Q.

Dr. Raynald Dery

1825 Des Roches St.

St. Pascal, Quebec 3, P.Q.

Dr. Paul.A. Deslippe

250 St. Georges

Laprairie, P.Q.

Dr. Simon Dombrowski

2960 Gentilly

Ste Foye, P.Q.

Dr. A. A. Dougan

33 Clough Ave.

Lennoxville, P.Q.

Dr. Arthur S. Douglass

Knowlton, P.Q. 
Dr. Gedeon Douville 1314 Lemoine

Sillery, P.Q.

Dr. Henri Drolet 49 St. Jean Baptiste Loretteville, P.Q.

Dr. Marius Dubeau Mattawa St., G. B. 78

Ferme Ste. Therese, R.R. 2

Ste. Rose, Laval Co., P.Q.

Dr. Antonio Duchesne

C. P. 115

Granby, P.Q.

Dr. Roland Duchesne

1284 Des Hospitalières

Sillery, P.Q.

Dr. Ladislas Dufour

82 Fraser

Rivière-du-Loup, P.Q.

Dr. Andre Dugas

Hôtel-Dieu, St. Valier

Chicoutimi, P.Q.

Dr. Yves Dugas

299 rue Notre Dame de Fatima

Duvernay, P.Q.

Dr. M. Dunkley

4652 Victoria Ave.

Montreal 29, P.Q.

Dr. Henri Durand

16 Sixth Ave.

Charlesbourg, P.Q.

Dr. Claude Duval

2240 Champfleury

Duvernay, P.Q.

Dr. Gladys Ellison

3460 Simpson St.

Montreal, P.Q.

Dr. Ural Erkal

Hôpital Général de Verdun

4000 Blvd. Lasalle

Montreal 19, P.Q.

Dr. Juan M. Escudero

80 Pine Ave. W., Apt. 207

Montreal, P.Q.

Dr. William J. Farley

3330 Ridgewood Ave., Apt. 44

Montreal, P.Q.
Dr. Robert Ferguson

739 Upper Belmont Ave.

Westmount, P.Q.

Dr. Hugues Ferland

2090 Rue Bedford

St. Bruno, Cté Chambly, P.Q.

Dr. John D. A. Firth

Apt. 33, 3644 Ontario Ave.

Montreal, P.Q.

Dr. Guy Fortin

215 S. Richelieu

St. Hilaire, P.Q.

Dr. Louns Fugere

27 Le Payeur

Ste Foy 10, P.Q.

Dr. A. Aimé Gagné

35 St. Francois, No. 2

Hull, P.Q.

Dr. Jean-Guy Gagné

3255 Lavallee

Ste Foy, Quebec 10, P.Q.

Dr. Rita Gagne-Desrosiers

6665-44th Ave., Apt. 4

Montreal 35, P.Q.

Dr. Roger Gagnon

504 Blvd. des Prairies

Laval des Rapides

Montreal 9, P.Q.

Dr. Paul Galıbois

355-22ième rue

Quebec, P.Q.

Dr. Albểrt Garand

5020 St. Kevin, App. 1

Montreal 29, P.Q.

Dr. Jean-Francois Garneau

6109-43 Ave.

Rosemont, Montreal 36, P.Q.

Dr. L. H. Gatien

3810 St. Joseph Blvd.

Lachine, P.Q.

Dr. P. H. Gauvin

7488 Ave. Abbe Trudel

Charlesbourg, P.Q.

Dr. Phillippe Gendron

St. Joseph Hospital

Three Rivers, P.Q. 
Dr. Gaston Giguère

St-Michel, Cté. de Bellechasse, P.Q.

Dr. Paul-Emile Gilbert

Hôtel-Dieu, St. Jerome

Cté. Terebonne, P.Q.

Dr. R. G. Gilbert

Montreal Neurological Institute

Montreal, P.Q.

Dr. Deirdre M. M. Gillies

Queen Elizabeth Hospital

2100 Marlowe Ave.

Montreal 28, P.Q.

Dr. Maurice Gladu

160 Heneker

Sherbrooke, P.Q.

Dr. Marcel Goyer

259 Moisan

Drummondville, P.Q.

Dr. Yvon Gravel

3586 Gertrude

Verdun, P.Q.

Dr. H. R. Griffith

3445 Northcliffe Ave.

Montreal, P.Q.

Dr. Roger Gaetan Guérin

149 de Normandie

St. Bruno, Cté. Chanbly, P.Q.

Dr. Roger Guerrette

40 Ave. Begon

Quebec 10, P.Q.

Dr. Edner Guerrier

3840 Rue St. Urbain

Montreal 18, P.Q.

Dr. Claude Guimond

Hôpital de l'Enfant Jésus

Quebec, P.Q.

Dr. Peter Gurrin

Montreal Neurological Institute

3801 University St.

Montreal, P.Q.

Dr. Jaime A. Gutierrez

2835 Goyer, Apt. 18

Montreal, P.Q.

Dr. Elzear Hamel

55 Church

Verdun, P.Q.
Dr. Albert Hamelin

1925 de la Ronde

Quebec, P.Q.

Dr. Paul Hamelin

2825 Boulevard du Carmel

Trois Rivières, P.Q.

Dr. Clement Harel

400 Fountainebleau

Duvernay, Montreal 40, P.Q.

Dr. Claude D. Hebert

Hôpital Laval

2725 Chemin Ste Foy

Quebec 10, P.Q.

Dr. M. Hoffman-Zukowski

126 rue Conrad

Laval des Rapides

Montreal 40, P.Q.

Dr. Jacques Houde

Hôtel-Dieu de Quebec

Quebec, P.Q.

Dr. Roland Hould

3520 Duvernay

Trois Rivières, P.Q.

Dr. Fernando Hudon

1601 Blvd. Laurier

Sillery, P.Q.

Dr. Raymond Hudon

G.P. 1323, R.R. 2

Fabreville, P.O.

Dr. Ali Ibrahim

Youville Hospital

Noranda, P.Q.

Dr. Marcel Jacob

5355 O'Bryan Ave., N.D.G.

Montreal, P.Q.

Dr. André Jacques

Dept. of Anaesthesia

L'Hôtel-Dieu de Québec

Palace Hill, Quebec, P.Q.

Dr. Alfred Jobidon

2352 Choquette

Quebec, P.Q.

Dr. André Joyal

1820 Blvd. St. Joseph E.

Montreal, P.Q. 
Dr. Michael Keeri-Szanto

2860 Cote de Liesse Rd.

Montreal 9, P.Q.

Dr. J.J. Kelly

423 Roslyn Ave.

Westmount, Montreal 6, P.Q.

Dr. David Korman

5585 Clanranald

Montreal, P.Q.

Dr. André Labrecque

2825 Sarrazin

Quebec 3, P.Q.

Dr. Joseph Lafortune

422 Manseau Blvd.

Joliette, P.Q.

Dr. Jean-Louis Lajoie

10432 Place Fleury

Montreal 12, P.Q.

Dr. Raymond L'Allier

8230 Champagneur

Montreal 15, P.Q.

Dr. Yves Lamarche

893 D'Iberville

Chicoutimi, P.Q.

Dr. Georges Lambert

1027 Commerciale

St. Romouald, P.Q.

Dr. J. J. Lambert

475 Wiseman Ave., Outremont

Montreal 8, P.Q.

Dr. Armand Lamontagne

Service d'Anesthésie, Enr.

1694 1st Ave.

Quebec, P.Q.

Dr. Louis Lamoureux

Hôpital Notre Dame

Montreal, P.Q.

Dr. Rouville Lamy

191 Sud Rue Brooks

Sherbrooke, P.Q.

Dr. Jean Langevin

404 Woodlea

Ville Mount Royal, P.Q.

Dr. Irenne Lapierre

1213 Commerciale

St. Romuald, P.Q.
Dr. Jean Laporte

415 Dobie Ave.

Town of Mount Royal, P.Q.

Dr. Eric W. Larking

Montreal General Hospital

Montreal, P.Q.

Dr. Patrick R. Laughrea

515 Fecteau

Thetford Mines, P.Q.

Dr. Jean Paul Lavallée

Hôpital Notre Dame

Montreal, P.Q.

Dr. Gerard Lavoie

Maisonneuve Hospital

Montreal, P.Q.

Dr. Anne B. Lawson

37 Church Hill

Montreal 6, P.Q.

Dr. Joseph Leavitt

4489 Maplewood Ave.

Montreal, P.Q.

Dr. Rene Lebeau

3725 Maplewood

Montreal, P.Q.

Dr. Etiennette Lebel

210 Est Fleury

Montreal, P.Q.

Dr. J. Jacques LeBlanc

27 Gendron St.

Hull, P.Q.

Dr. Claude Leduc

5753 Northmount

Montreal 26, P.Q.

Dr. Lionel J. Leduc

2160 Capitaine Beriner

Bordeaux, Montreal, P.Q.

Dr. G. A. Lefebvre

33 rue des Rois

Beaucharnois, P.Q.

Dr. Maurice Legare

2171 Sherbrooke St. E.

Montreal, P.Q.

Dr. L. Legault

300 Carré St. Louis

Montreal 18, P.Q. 
Dr. Jean-Denis Lemay

226 Mont-Plaisant

Sherbrooke, P.Q.

Dr. Richard Lemieux

Buckingham, P.Q.

Dr. Claire Lemire

Hôpital Notre Dame

1560 Sherbrooke E.

Montreal, P.Q.

Dr. Claude Lepage

4591 d'Orleans

Montreal 36, P.Q.

Dr. F. Lepinskie

5559 Oakwood Ave.

Montreal 29, P.Q.

Dr. René Létienne

c/o Clinique Medicale du Parc Lafontaine

1657 Rue du Parc Lafontaine

Montreal 34, P.Q.

Dr. Marcel L'Etoile

2289 Rue Viel

Montreal 12, P.Q.

Dr. Armand Levac

4395 Ave. Verdun

Montreal, P.Q.

Dr. Abraham Levitz

5628 Pinedale Ave., Côte St. Luc

Montreal, P.Q.

Dr. G. A. Lewis

12 Lakeshore Rd.

Beaconsfield, P.Q.

Dr. Leon Longtin

45 Hazelwood

Outremént, P.Q.

Dr. Edouard Lortie

6531 Molson St.

Montreal, P.Q.

Dr. Jules E. Lupien

10842 L'Esplanade

Montreal, P.Q.

Dr. R. Lussier

3814 Grey, N.D.G.

Montreal, P.Q.
Dr. D. W. MacMillan

1165 Maple Ave.

Quebec 6, P.Q.

Dr. David J. L. MacWatt

38 Thornton Ave.

Town of Mount Royal, P.Q.

Dr. Jean-Guy Maill

11239 Drouart

Montreal 12, P.Q.

Dr. Paul Marcoux

298 rue Labelle

St. Jerome, Co. Terrebonne, P.Q.

Dr. R. L. Marin

127 Council

Sherbrooke, P.Q.

Dr. A. Marsolais

35 Ave. Jasper

Montreal 16, P.Q.

Dr. Emile Martimbeau

3165 Rue Tremblay

Montreal, P.Q.

Dr. William W. Martin

1125 Monctea Ave.

Quebec, P.Q.

Dr. Victorin Masson.

70 Berthiaume

Ste Foy, P.Q.

Dr. Roger Matte

Hôpital du St. Sacrement

1050 Chemin Ste. Foy

Quebec 6, P.Q.

Dr. Alexander Matzko

6702 Des Erables Ave.

Montreal 35, P.Q.

Dr. Andre McClish

2121 Rue Richer, Apt. 7

Ste Foye, Quebec 10, P.Q.

Dr. Alexander W. McDonald 4665 Prince of Wales Ave.

Montreal 28, P.Q.

Dr. John J. McGrath

4840 Ave. Bourret, Apt. 25

Montreal 26, P.Q.

Dr. Clarice M. McKiernan-Lavoie

Maisonneuve Hospital

Montreal, P.Q. 
Dr. P. McLaughlin 7045 St. Denis St. Montreal, P.Q.

Dr. P. McMahon 166 King St. W. Sherbrooke, P.Q.

Dr. Jean-Paul Methot 12260 Blvd. Taylor Montreal, P.Q.

Dr. Gerard G. Mignault 12485 Place St. Castin Montreal 12, P.Q.

Dr. Velta K. Mikelsons 3768 Botrel Ave.

Montreal, P.Q.

Dr. Rosaire Milette 200 Ste. Damasse Drummondville, P.Q.

Dr. Nadia Mistruzzi 80 Pine Ave. W., Apt. 404 Montreal 18, P.Q.

Dr. Claude Mitton Hôpital Général de Verdun 4000 Blvd. LaSalle Verdun, P.Q.

Dr. Andre L. Moise

Hôpital Notre Dame 1560 Sherbrooke E. Montreal, P.Q.

Dr. D. A. Mondor 4902 Verdun Ave. Verdum, P.Q.

Dr. Lasalle Mondor C.P. 507 Grand'Mere, P.Q.

Dr. Yvon Morinville Louiseville, P.Q.

Dr. Mary Morris 1 Ellerdale Rd., Apt. 27 Hampstead 29, P.Q.

Dr. A. N. Mungall 3421 Drummond St., Apt. 97 Montreal, P.Q.
Dr. Olga S. Nadiak Montreal General Hospital Dept. of Anaesthesia Montreal 2, P.Q.

Dr. A. B. Noble 316 Lethbridge Ave.

Town of Mount Royal, P,Q.

Dr. J. Ovila Noel

301 Council

Sherbrooke, P.Q.

Dr. Peter E. O'Shaughnessy

3198 Lacombe

Montreal 26, P.Q.

Dr. Edward Osmolski 11055 Pasteur St.

Montreal, P.Q.

Dr. Paul E. Otton

Royal Victoria Hospital

Montreal 2, P.Q.

Dr. Camille Ouellet

171 Hickson

St. Lambert, P.Q.

Dr. Paul Ouellet

2095 lère Ave.

Quebec, P.Q.

Dr. Bernard Paiement

5822 Roi Rene

Ville D'Anjou, P.Q.

Dr. Kris Panwar

1578 McGregor St.

Montreal 25, P.Q.

Dr. Pierre Paquet Hôtel-Dieu Hospital Jonquierre, Kenogami, P.Q.

Dr. Bernard Paradis

2110 Bourbonniere

Quebec, P.Q.

Dr. Francois Parant 83 Bon Air, Ste Foy Quebec 10, P.Q.

Dr. Jean-Guy Patoine 166 St. François E. Quebec, P.Q. 
Dr. Wilfrid Pellerin 176 Rue Sherbrooke Magog, P.Q.

Dr. Jacques Pelletier 429 rue de la Vigerie Quebec 10, P.Q.

Dr. Loyola Perras 530 Grosvenor Westmount, P.Q.

Dr. Luc Perreault 2407 Victore-Doré Montreal, P.Q.

Dr. Paul Perron 2915 Maplewood, Apt. 505

Montreal 26, P.Q.

Dr. Anthony Petrasek 1948 Norway Rd.

Town of Mount Royal, P.Q.

Dr. Jean Phaneuf

C.P. 102, St. Hyacinthe, P.Q.

Dr. Gilles Piche

Arthabaska, P.Q.

Dr. Andree Pinault

843 Riverside Dr.

St. Lambert, P.Q.

Dr. Yves E. Piuze

970 Clement

Ville St. Laurent, P.Q.

Dr. Marcel Plamondon

1280 Patenaude

Quebec, P.Q.

Dr. Paul Plourde

Hôpital Hôtel-Dieu

Montmagny, P.Q.

Dr. Florian Poirier

326 Ste. Marie

Jonquiere, P.Q.

Dr. Jacques Poliquin

10 Rue Jacques, Terrace Bon Air Quebec 7, P.Q.

Dr. Ph. Poliquin

Maniwaki, Gatineau County, P.Q.
Dr. J. Claude Pouliot 2979 Matapedia

Ste. Foy, Quebec 10, P.Q.

Dr. David J. Power 4131 Harvard Ave., N.D.G.

Montreal, P.Q.

Dr. Guy Preville

535 Lafleur

Lasalle, P.Q.

Dr. Ralph L. Prime

463 Hudson Ave.

Montreal West, P.Q.

Dr. Hortense Prud'homme

131 Graham Blvd.

Montreal, P.Q.

Dr. Paul Quan

Montreal Children's Hospital

Montreal 25, P.Q.

Dr. Jean Quintal

77 Rue Brock

Drummondville, P.Q.

Dr. Ferenc Racz

Box 866

La Sarre, P.Q.

Dr. J. A. Remy

385 Woodlea

Ville Mont-Royal, Montreal, P.Q.

Dr. Jean Rheault

3615 Ridgewood, App. 402

Montreal 26, P.Q.

Dr. Lucien Rinfret

1045 Ave. Begin

Quebec 6, P.Q.

Dr. R. W. Roberts

2126 Norway Rd.

Town of Mount Royal, P.Q.

Dr. Francis A. Robillard

Montreal General Hospital

Montreal, P.Q.

Dr. J. Maurice Robillard

4000 Ave. Marlowe

Montreal 28, P.Q. 
Dr. J. Gordon Robson

301 Victoria Dr.

Baie D'Urfée, P.Q.

Dr. Alexander Romagnoli

44 Highfield Ave.

Montreal 16, P.Q.

Dr. Jose K. Rosales

64 Banstead Rd.

Montreal West, P.Q.

Dr. J. M. Rouleau

St. Gregoire Co.

Nicolet, P.Q.

Dr. C. A. Roussin

131 North St. Charles

Joliette, P.Q.

Dr. C. E. Roy

Hôpital St. Luc, 1058 St. Denis

Montreal, P.Q.

Dr. Paul E. Roy

P.O. Box 670

Cowansville, P.Q.

Dr. Lea Rubinyi

6417 Lennox

Montreal, P.Q.

Dr. D. Saindon

C.P. 116, Rivière-du-Loup, P.Q.

Dr. O. Schulte-Steinberg

1420 Pine Ave. W., Apt. 19

Montreal, P.Q.

Dr. Gerard Seguin

6 Molson

Valleyfield, P.Q.

Dr. C. A. Sheridan 1960 Laird Blvd.

Montreal 16, P.Q.

Dr. F. X. Simard

865 D'Iberville

Chicoutimi, P.Q.

Dr. Gaston Simard

130 Champlain

Baie Comeau, P.Q.
Dr. A. V. Simon

4865 Queen Mary Rd.

Montreal, P.Q.

Dr. Neeranjan Singh

P.O. Box 962, Station B

Montreal 2, P.Q.

Dr. Paul E. Sirois

16 Lacasse

Hull, P.Q.

Dr. H. M. Slater

5045 Prince of Wales Ave.

Montreal, P.Q.

Dr. H. G. Smith

745-5th Rue

Shawinigan Falls, P.Q.

Dr. Y. Sormany

I031 Desaulniers

Chicoutimi, P.Q.

Dr. Shena R. Sourkes

5235 St. Ignatius Ave.

Montreal, P.Q.

Dr. Benigno M. Sulit, Jr.

Hôpital du Sacre-Coeur

5400 Ouest Blvd. Gouin

Montreal 9, P.Q.

Dr. John B. I. Sutherland

450 Elm Ave.

Westmount 6, P.Q.

Dr. F. Tanquay

100 Murray

Sherbrooke, P.Q.

Dr. J.-Alexandre Tardif

205 de la Cathédrale

Rimouski, P.Q.

Dr. Elie Therrien

10007 St. Denis

Montreal, P.Q.

Dr. A. Tiltins

L'Hôpital Ste-Justine pour les Enfants

Montreal, P.Q.

Dr. Jean-Pierre Tousignant

5489 rue Mignault

Montreal, P.Q.

Dr. Michel Tousignant

566 Hôtel Dieu St., Apt. 6

Chicoutimi, P.Q. 
Dr. Maurice L. Trahan

4967 Coronation, N.D.G.

Montreal, P.Q.

Dr. Lionel Tremblay

3840 Wilson

Montreal 28, P.Q.

Dr. Andre C. Trempe

5020 St. Kevin, Apt. 1

Montreal 29, P.Q.

Dr. Davy Trop

1560 Sherbrooke E.

Montreal, P.Q.

Dr. Yvon Turmel

2579 Mousseau

Montreal 5, P.Q.

Dr. Hugues Turnier

5740 Northmount

Montreal, P.Q.

Dr. Annegnet Uhthoff

498-38th Ave.

Ville Lasalle, Montreal, P.Q.

Dr. W. A. Urbanski

3330 Bedford, Apt. 31

Montreal 26, P.Q.

Dr. Reza Vazirnia

3734 Cote St. Catherine Rtd., No. 15

Montreal 26, P.Q.
Dr. Gabriel Veilleux

Hôpital Notre Dame

Montreal, P.Q.

Dr. Romeo Veilleux

208 King St. E.

Sherbrooke, P.Q.

Dr. Jean Vigneau

230 Mercille Ave.

St. Lambert, P.Q.

Dr. Pat Vigneault

11470 rue Drouart

Montreal 12, P.Q.

Dr. Remigio C. Villaricc

3380 Maplewood Ave., Apt. 104

Montreal 26, P.Q.

Dr. Dagmar Vosu

Catherine Booth Maternity Hospital

440 Walkley

Montreal, P.Q.

Dr. W. A. Wielhorski

c/o Maisonneuve Hospital

Montreal, P.Q.

Dr. F. R. H. Wrigley

7421 Bayard St.

Montreal 16, P.Q.

Dr. John E. Wynands

22 Fairfield Cres.

Montreal West, P.Q.

New Brunswick Division

Dr. Margaret N. Barr

c/o St. John General Hospital

St. John, N.B.

Dr. A. M. R. Brown

15 Coburg St.

St. John, N.B.

Dr. Jean Caron

532 Princess Dr.

Bathurst, N.B.

Dr. F. R. Connell

177 Princess St.

St. John, N.B.
Dr. Egbert Daigle

10 Hawker Ave.

Moncton, N.B.

Dr. J. Daigle

Dalhousie, N.B.

Dr. E. R. Davis

17 Sixth St.

St. John, N.B.

Dr. Claude Desjardins

Petit-Rocher, N.B. 
Dr. Stanislaw B. Donigiewicz P.O. Box 638

Antigonish, N.S.

Dr. D, Drury

156 Victoria St.

Amherst, N.S.

Dr. Alan A. Drysdale

34 Armerescent $W$.

Halifax, N.S.

Dr. R. A. P. Fleming 361 Crows Nest Dr.

Halifax, N.S.

Dr. Norman G. Glen

Amherst, N.S.

Dr. G. L. Goulding

Victoria General Hospital

Halifax, N.S.

Dr. Donald V. Graham

58 Norwood St.

Halifax, N.S.

Dr. Robert N. Hetherington

P.O. Box 54

R.R. 1, Greenwood Hts.

Armdale, N.S.

Dr. A. S. MacIntosh

313 Spring Garden Rd.

Halifax, N.S.

Dr. E. J. MacLeod

8 Glenwood Ave.

Dartmouth, N.S.
Dr. Roberta Nichols 1058 Tower Rd.

Halifax, N.S.

Dr. A. F. Pasquet

6010 Inglis St.

Halifax, N.S.

Dr. Ian E. Purkis

812 Young St.

Halifax, N.S.

Dr. Hadsel G. Qưigley

37 Bellevue Ave.

Halifax, N.S.

Dr. Joseph A. Roach

Acadia Ave.

New Waterford, N.S.

Dr. R. Gordon Simpson

26 Rigby Rd.

Sydney, N.S.

Dr. John A. Smith.

Windsor, N.S.

Dr. C. C. Stoddard

6533 Coburg Rd.

Halifax, N.S.

Dr. A. S. Wenning

5880 Chain Rock Dr.

Halifax, N.S.

Dr. L: J. Wiseman

Victoria General Hospital

Halifax, N.S.

Dr. A. C. Yelland

38 Murrayhill Dr.

Dartmouth, N.S.

\section{Prince Édward Island Divisicn}

Dr. C. M. Dewar

O'Leary, P.E.I.

Dr. Gilbert J. Gallant

281 Schurman Ave.

Summerside, P.E.I.
Dr. Douglas A. C. MacDonald

137 Nassau St.

Charlottetown, P.E.I.

Dr. L. E. Prowse

29 Greenfield Ave.

Charlottetown, P.E.I. 


\section{Newfoundland Division}

Dr. Raymond L. Bugden

38 Brookfield Ave.

Cornerbrook, Nfld.

Dr. Richard C. Condon

Medical Centre

Cornerbrook, Nfld.

Dr. Raymond A. Duffy

c/o Nfld. Anaesthesia Associates

95 LeMarchant Rd.

St. John's, NAd.

Dr. Charles U. Henderson

Waterford Bridge Rd.

St. John's, Nfld.

Dr. C. D. Kean

17 Topsail Rd.

St. John's, Nfld.

Dr. John James Kennedy

188 LeMarchant Rd.

St. John's, Nfld.
Dr. Robert Macdonald

3 Roche St.

St. John's, Nfld.

Dr. Irineo M. Nacino

General Hospital

St. John's Nfld.

Dr. A. Pace-Floridia.

180 LeMarchant Rd.

St. John's, Nfld.

Dr. Hazel N. Peisley

Apt. 50, Churchill Square Apts.

St. John's, Nfld.

Dr. Edward L. Sharpe

Grand Falls Clinic

Grand Falls, Nfld.

Dr. Thomas G. Stentaford

11 Winter Ave.

St. John's, Nfld.

US.A.

Dr. Geordis M. Aasheim

10325-204th Place S.W.

Edmonds, Wash., U.S.A.

Dr. John Abajian

38 Elsom Parkway

South Burlington

Vt., U.S.A.

Dr. David Allan

Director of Anaesthesia

Children's Memorial Hospital

707 Fullerton Ave.

Chicago 14, Ill., U.S.A.

Dr. James S. Barr

13B Medical Ct.

Greenville, S.C., U.S.A.

Dr. William H. Blair

1425 Lombardy Rd.

Pasadena, Calif., U.S.A.

Dr. A. K. Bradshaw

10030 Briarwood Lane

Mercer Island, Wash., U.S.A.
Dr. Maria Caldini

$116 \mathrm{f}$ Cherry St.

Denver 20, Colo., U.S.A.

Dr. Robert H. Cole

1534 Coventry Rd.

Charlotte 7, N.C., U.S.A.

Dr. Adeline Comeau

523 N. 15th St.

Clarinda, Iowa, U.S.A.

Dr. P. W. Crookes

Richmond Hospital

23rd St. and Gaynor Ave.

Richmond, Calif., U.S.A.

Dr. James $\mathbb{F}$. Cụmming

707 St. Croix St.

Hudson, Wis., U.S.A.

Dr. John I. Davies

University of Kansas Medical Center

Rainbow Blvd. at 39th St.,

Kansas City 12, Kan., U.S.A. 
Dr. Oswald S. Dawkins

Box 30,

Thompsonville, Mich., U.S.A.

Dr. Allan B. Dobkin

Dept. of Anaesthesiology

University Hospital

Syracuse, N.Y., U.S.A.

Dr. David Drabinsky

1707 S. Holt Ave.

Los Angeles 35, Calif., U.S.A.

Dr. C. F. Egan

Wyandotte General Hospital

Wyandotte, Mich., U.S.A.

Dr. Lionel Glassman

3615 Anton Farms Rd.

Baltimore 8, Md,, U.S.A.

Dr. Ian S. H. Harper

2217 Webster St.

San Francisco 15, Calif., U.S.A.

Dr. Ann B. Henschel

4709 N. Cramer St.

Milwaukee 11, Wis., U.S.A.

Dr. A. Hilado Jr.

1312 Gordon Rd.

Lyndhurst 24, Ohio, U.S.A.

Dr. Eric B. Howell

4208 Warrensville Centre Rd.

Warrensville Hts. 22, Ohio, U.S.A.

Dr. Elio Iannuzzi

45 Ryefield Road

Fitchburg, Mass.

Dr. Robert R. Jones

Mayo Clinic

Rochester, Minn., U.S.A.

Dr. Wm. P. G. Jones

St. Francis Hospital

3237 S. 166th St.

Milwaukee 15, Wis., U.S.A.

Dr. Gerard R. F. Landry

561 Cogleywood Lane

Council Bluffs, Iowa, U.S.A.
Dr. Audrey A. Lewis

Interns' Residence

The Children's Hospital Medical Center

300 Longwood Ave.

Boston, Mass., U.S.A.

Dr. Evan A. C. Lloyd

Veterans Administration Hospital

Durham, N.C., U.S.A.

Dr. Stewart T. Love

502 Laurel Ave.

Pennrock, Wilmington, Delaware, U.S.A.

Dr. Cyril R. MacLean

817 Waldo Blvd.

Manitowoc, Wis., U.S.A.

Dr. Horacio Martinez

2311 South Overlook Rid.

Cleveland, Ohio, U.S.A.

Dr. Johin A. McConnell

c/o Childien's Hospital of L.A.

4614 Sunset Blvd.

Los Angeles 27, Calif., U.S.A.

Dr. Elizabeth M. Martin

132 Oakly Rd.

Wooster, Ohio, U.S.A.

Dr. Gerda Menetrez

4201 Massachusetts Ave. N.W.

Washington 16, D.C., U.S.A.

Dr. Nguten Khac Minh

Box 31, University Hospital

Minneapolis 14, Minn., U.S.A.

Dr. Emerson A. Moffitt

1218 6th St. S.W.

Rochester, Minn., U.S.A.

Dr. Anthony J. O'Keefe

3721 N. 28th St.

Tacoma 7, Wash., U.S.A.

Dr. Robert Orr

1248 Waverly Place

Schenectady 8, N.Y., U.S.A.

Dr. Arthur J. Oswald

1961 Fairweather Rd.

Santa Ana, Calif., U.S.A. 
Dr. Edward Petrovich

2111 Ganyard Rd.

Akron 13, Ohio, U.S.A.

Dr. Imbi Ralyea

16645 Freeland

Detroit 35, Mich., U.S.A.

Dr. James Rintoul

281 Grosvenor Rd.

Rochester 10, N.Y., U.S.A.

Dr. James M. G. Ritchie

724 Niles Ave.

Everett, Wash., U.S.A.

Dr. M. J. Roebuck

629 Kappock St., Apt. 7H

Bronx, N.Y., U.S.A.

Dr. Wm. Armin Seibert, Jr.

251-26 Gaskell Rd.

Little Neck, Long Island, N.Y., U.S.A.

Dr. C. R. Stephen

Duke University

Durham, N.C., U.S.A.

Dr. S. H. Stolar

426 Medical Center Rd., Apt. A-202

Morgantown, W. Va., U.S.A.
Dr. M. A. Tanasichuk

527 S.E. 5th St.

Minneapolis 14, Minn., U.S.A.

Dr. James V. Taylor

3301 N.E. 73rd St.

Seattle 15, Wash., U.S.A.

Dr. Roger J. Thompson

92 Henderson Dr.

Penfield, N.Y., U.S.A.

Dr. Peter C. Volshin

4725 Daleridge Rd.

La Canada, Calif., U.S.A.

Dr. E. H. Watts

6226 N. Mockingbird Lane

Scottsdale, Ariz., U.S.A.

Dr. Hugh D. Westgate

5818 Sunset Dr.

Hopkins, Minn., U.S.A.

Dr. James K. Whittal

6521 27th N.E.

Seattle 15, Wash., U.S.A.

\section{Other Countries}

Dr. Eileen M. Dixon

Laurel Hill Horden

County Durham, England

Dr. F. C. Emberton

Clinical Research Dept., Pfizer Ltd.

Sandwich, Kent, England

Dr. S. A. Fleming

University of Lagos Medical School

Private Mail Bag 12003

Surulere, Lagos, Nigeria, West Africa

Dr. C. Langton Hewer

33 Stormont Rd., Highgate

London N.6, England

Dr. R. B. Irwin

Surgeon Commander

No. 3 "F" Wing R.C.A.F., CAPO 4055

Canadian Armed Forces, Europe
Mr. A. Charles King

27 Devonshire St.

London W.1, England

Professor Sir Robert Macintosh

Pembroke College

Oxford, England

Dr. Geoffrey Organe

17 Burghley Rd., Wimbledon

London S.W.19, England

Dr. Mukteshwar Prosad

Darbhanga Pálace Rd.

Chouhatta, Patna 4

Bihar, India

Dr. E. S. Russell

University of Lagos Medical School

Private Mail Bag 12003

Surulere, Lagos, Nigeria, West Africa 
Dr. Guy Screech

Penang General Hospital

Panang, Malaya

Di. Edward T. Thomas

St. James' Hospital, Balham

London S.W.12, England
Major G. A. VanVliet

1 C.B.M.U., CAPO 5050

Canadian Armed Forces, Germany

Dr. Anthony C. Webster

63 The Balk

Walton, Nr. Wakefield

Yorks., England 